\title{
Construindo um modelo de sistema de cuidados*
}

\author{
Developing an explanatory theoretical model of system of care
}

Construyendo un modelo de sistema de cuidados

\section{Alacoque Lorenzini Erdmann', Francisca Georgina Macêdo de Sousa², Dirce Stein Backes ${ }^{3}$, Ana Lúcia Schaefer Ferreira de Mello}

\section{RESUMO}

Objetivo: Construir um modelo teórico explicativo de Sistema de Cuidados. Metodo: Estudo exploratório qualitativo apoiado nos pressupostos da Grounded Theory. Participaram do estudo 15 profissionais e os dados foram coletados por entrevista aberta. Resultados: O Modelo teórico foi delimitado a partir do fenômeno Construindo o Sistema Complexo de Cuidados determinado pelas categorias: Convivendo com a dinamicidade do sistema de cuidados e Organizando o sistema de cuidados a partir de múltiplas interações. Conclusão: O modelo de sistema de cuidados apresentou-se como sistema vital e dinâmico construído a partir de redes interacionais de vários saberes do agir humano expresso pelo trabalho compartilhado, multidisciplinar e em equipe.

Descritores: Atenção à saúde; Cuidado centrado no paciente; Administração dos cuidados ao paciente; Modelos de enfermagem

\begin{abstract}
Objective: To develop an explanatory theoretical model of the system of care. Method: Grounded theory served as the conceptual framework to conduct this qualitative exploratory study. Unstructured interviews were conducted among 15 care professionals. Results: The theoretical model was delimited from the phenomenon "building complex system of care" as determined by the following categories: "dealing with a dynamic system of care" and "organizing the system of care through multiple interactions." Conclusion: The explanatory theoretical model of system of care was developed as a vital and dynamic system built from interactions among health care professional who share responsibilities and ways of knowing through multidisciplinary work.
\end{abstract}

Keywords: Health care (Public Health); Patient-centered care; Patient care management; Models,nursing

\section{RESUMEN}

Objetivo: Construir un modelo teórico explicativo de Sistema de Cuidados. Método: Se trata de un estudio cualitativo exploratorio apoyado en las premisas de la Teoría Fundamentada en los Datos. Participaron del estudio 15 profesionales. Los datos fueron recolectados por medio de una entrevista abierta. Resultados: El modelo teórico se delimitó al fenómeno Construyendo el Sistema Complejo de Cuidados determinado por las categorías: Viviendo junto con la dinamicidad del sistema de cuidados y Organizando el sistema de cuidados a partir de interacciones múltiples. Conclusión: El modelo de sistema de cuidados se presenta como un sistema vital y dinámico construido a partir de redes de interacción de conocimientos del actuar humano expresado en el trabajo conjunto, multidisciplinario y en equipo.

Descriptores: Atención a la salud; Atención dirigida al paciente; Manejo de la atención al paciente; Modelos de enfermería

\footnotetext{
* Trabalho derivado do Projeto de Pesquisa intitulado "A complexidade do sistema organizacional de cuidados de enfermagem: práticas dos serviços de saúde em ambiente mais saudável" desenvolvido pelo Grupo de Estudos e Pesquisas em Administração de Enfermagem e Saúde - GEPADES/UFSC.

1 Doutora em Filosofia da Enfermagem; Professora Titular da Universidade Federal de Santa Catarina - UFSC - Florianópolis (SC), Brasil.

2 Pós-graduanda do Programa de Pós-Graduação em Enfermagem da Universidade Federal de Santa Catarina - UFSC - Florianópolis (SC), Brasil; Docente do Curso de Enfermagem - UFMA,

${ }^{3}$ Pós-graduanda de Enfermagem pelo Programa de Pós-Graduação da Universidade Federal de Santa Catarina - UFSC - Florianópolis (SC), Brasil

${ }^{4}$ Doutora em Enfermagem pelo Programa de Pós-Graduação em Enfermagem da Universidade Federal de Santa Catarina - UFSC - Florianópolis (SC), Brasil.
} 


\section{INTRODUÇÃO}

\section{Aproximando-nos dos significados de sistema de cuidados}

Tradicionalmente, sob o enfoque mecanicista e simplificado de apreender o cuidado, a pessoa humana, freqüentemente, é concebida em partes/fragmentos. No entanto, novos referenciais vêm, atualmente contribuir para a compreensão do cuidado enquanto um sistema complexo $^{(1)}$, isto é, enquanto um fenômeno vital, dinâmico e essencial na vida dos seres e da natureza. $\mathrm{O}$ sistema de cuidados em saúde afirma-se como "disposição relacional" (2) e caracteriza-se como unidade complexa que liga, transforma, mantém ou produz acontecimentos, componentes e indivíduos. Enquanto disposição relacional, a organização do sistema de cuidados remete ao plano dinâmico da interação, não podendo ser reduzida à ordem, embora a comporte e a produza $^{(2)}$. Pensar em sistema de cuidados é apontar para a auto-organização desse sistema, considerando aspectos como autonomia, individualidade, relações e atitudes profissionais. Tais circunstâncias nos remetem a aceitar as incertezas, as ambivalências e as contradições presentes no sistema de cuidados para que seja possível lidar com a complexidade do real. Focalizar o sistema de cuidados nesta perspectiva significa produzir um conhecimento complexo em saúde, e compreender o cuidado como sistema vital e dinâmico que implica na construção de redes não lineares. Nesse sentido, o sistema de cuidados à saúde/doença é um coletivo constituído pela totalidade das práticas, das atitudes e do conhecimento dos vários profissionais que dão sustentação à dinâmica do cuidado. Esta perspectiva nos remete ao princípio hologramático, em que "o todo está na parte que está no todo, e a parte poderia estar mais ou menos apta a regenerar o todo"(3). Assim, a complexidade organizacional do todo necessita da complexidade das partes, a qual necessita retroativamente da complexidade organizacional do todo. Mas, qual seria a compreensão de sistema de cuidados para os profissionais de saúde? Que significados os profissionais atribuem a sistema de cuidados? A partir destes questionamentos procuramos compreender sistema de cuidados no processo saúdedoença centrando na pessoa humana e no cuidado em saúde. A partir destas reflexões, foi definido como objetivo do estudo, construir um modelo teórico explicativo sobre o significado de Sistema de Cuidados para um grupo de profissionais da saúde.

Buscando caminhos metodológicos para a compreensão do significado de sistema de cuidados para os profissionais da saúde

O estudo é do tipo exploratório descritivo com abordagem qualitativa dos dados coletados em duas unidades hospitalares de duas cidades, uma no sul e outra no nordeste do Brasil, no período de janeiro a março de 2006. A entrevista aberta foi utilizada como instrumento para coleta dos dados norteada pela pergunta "Como você compreende um sistema de cuidados?". As entrevistas foram gravadas em meio digital e, depois de transcritas, analisadas considerando os pressupostos da Grounded Theory ou Teoria Fundamentada nos Dados - TFD ${ }^{(4-8)}$. Participaram do estudo 15 profissionais da saúde que desenvolviam atividades no contexto hospitalar selecionados de forma intencional. A amostragem teórica na TFD é parte do processo de coleta de dados, cujo objetivo consiste em identificar pessoas, acontecimentos e locais que potencializam as oportunidades de descobrir variações entre conceitos, densificando as categorias, suas propriedades e dimensões ${ }^{(4)}$.

A amostragem teórica compreende a etapa em que o pesquisador decide quais informações são necessárias e onde encontrá-las, direcionando os questionamentos aos temas emergentes e importantes para o estudo. A saturação teórica ocorre quando análises adicionais não mais contribuem para descobrir algo novo sobre uma categoria ${ }^{(5)}$. Para atender a estes pressupostos, os sujeitos foram agrupados em quatro grupos amostrais. O primeiro grupo foi constituído por cinco profissionais de saúde e o objetivo da coleta foi obter respostas sobre os significados de sistema de cuidados para estes profissionais. A partir da análise dos dados brutos e da construção dos primeiros códigos, os significados de sistema de cuidados foram delineados orientando-se no sentido da humanização do cuidado e da interdisciplinaridade. Estes dados nos direcionaram para um outro questionamento que nos possibilitou a seguinte hipótese: os profissionais de saúde atribuem o trabalho interdisciplinar e a valorização do ser humano como significados para o sistema de cuidados?

Assim, o segundo grupo amostral, foi composto por quatro profissionais, duas enfermeiras assistenciais, uma gerente de enfermagem e uma assistente social de uma unidade de alta complexidade. O objetivo da investigação, neste grupo, foi compreender como as ações interdisciplinares e o cuidado humano permeavam o significado de sistema de cuidados para estes profissionais. $\mathrm{Na}$ seqüência, direcionamos a entrevista, com a seguinte pergunta: Que atitudes/práticas são experenciadas por vocês para o cuidado em ambiente de alta complexidade? Com estes dados, analisados e comparados aos anteriores, percebemos que o sistema de cuidados estava sendo apresentado como um sistema complexo, no qual permeavam atitudes e práticas de vários profissionais, sobretudo em uma situação de rompimento com o modelo biomédico e de reconhecimento da integralidade do cuidado. Neste momento, sentimos a necessidade de compreender como os profissionais se organizavam para 
o cuidado integral, em que saberes e valores baseavam as suas práticas. Conduzimos-nos, desta vez, ao serviço de clínica médica e cirúrgica, envolvendo quatro novos profissionais. A pergunta nesta etapa foi: Que experiências vocês vivenciam no cuidado hospitalar? Este grupo apresentou algumas especificidades em relação aos grupos anteriores, apontando dificuldades e desafios no cuidado referentes à organização do sistema, à interação da equipe e a resolutividade dos serviços. Novas hipóteses foram formuladas e, a partir delas, buscamos respostas sobre os significados de sistema de cuidados no contexto hospitalar, retornando aos dados brutos, codificando e construindo o fenômeno central. O quarto grupo amostral, constituído por dois profissionais, teve como objetivo, portanto, validar o modelo teórico cujo foco foi o trabalho interdisciplinar em um sistema de cuidados.

$\mathrm{Na}$ TFD, a coleta e análise dos dados são processos que acontecem concomitantemente. A pesquisa, então, tem a sua continuidade no momento em que são definidas categorias de análise que constituem os componentes do modelo. As categorias são organizadas e articuladas para a formulação e integração do modelo teórico explicativo do fenômeno em questão.

Quanto aos aspectos éticos, o projeto foi avaliado pelo Comitê de Ética e Pesquisa da Santa Casa de Misericórdia de Pelotas - RS, em reunião realizada no dia 07.12.05 com parecer favorável para realização do estudo. As pesquisadoras procuraram obedecer a todos os aspectos éticos que envolvem a pesquisa com seres humanos e a coleta de dados só foi iniciada após parecer do Comitê e assinatura do Termo de Consentimento Livre e Esclarecido pelos informantes.

O estudo foi guiado pelos fundamentos teóricometodológicos da TFD que é uma metodologia de investigação baseada na formação ordenada de uma teoria a partir dos dados encontrados por meio da pesquisa social $^{(5)}$. A teoria deve explanar, claramente, categorias e hipóteses de maneira que possam ser verificadas em pesquisas presentes e futuras. Além disso, deve ser compreensível e ter aplicação prática ${ }^{(6)}$. Uma teoria que preenche esses requisitos deve ser adaptável ao tipo de situação em que está sendo pesquisada e ser funcional, quando colocada em uso. $\mathrm{O}$ atributo da adaptabilidade quer dizer que as categorias devem ser emergentes, não forçadas, ou seja, devem ser a expressão abstrata dos dados. Já o atributo da funcionalidade implica ser a teoria significativamente relevante e capaz de explicar, predizer e interpretar o fenômeno estudado ${ }^{(6)}$. Um outro requisito é a capacidade de modificação da teoria à medida que surgem novos dados ${ }^{(6)}$. O papel do pesquisador, entretanto, é desenvolver uma "adaptação emergente" entre os dados e categorias pré-existentes, garantindo que estas continuem funcionais ${ }^{(7)}$. A estratégia metodológica utilizada para a descoberta de uma teoria que se fundamenta em dados é a análise comparativa, e, por meio deste processo dois tipos de teorias são gerados: substantivas e formais ${ }^{(5)}$. Teorias substantivas são teorias desenvolvidas para uma área da investigação sociológica empírica e substantiva como relações de raça, cuidado de pacientes, educação profissionalizante, delinqüência, ou pesquisa organizacional. Teorias formais são desenvolvidas para uma área de investigação sociológica formal, conceitual, como estigmas, comportamentos desviados, organização formal, socialização, autoridade e poder ou mobilidade social ${ }^{(5)}$. Os elementos da teoria ou modelo teórico gerado por meio da análise comparativa e pela sensibilidade teórica do pesquisador são, primeiramente, categorias e suas propriedades conceituais, seguidas de hipóteses e relações generalizadas entre estas categorias e propriedades ${ }^{(5)}$. Construir uma teoria apoiada na TFD significa reduzir dados de muitos casos a conceitos, convertendo-os num conjunto de afirmações que explicam, em sentido geral, o que está acontecendo ${ }^{(4)}$. A categoria central representa o tema central da investigação e tem o poder de integrar a teoria ${ }^{(5)} \mathrm{com}$ as seguintes características ${ }^{(8)}$ : todas as categorias devem relacionar-se a ela; deve aparecer com freqüência nos dados; a explicação que se desenvolve a partir da relação entre as categorias deve ser lógica, consistente e emergente; a frase utilizada para descrevêla deve ser abstrata o bastante para que possa ser utilizada em outras áreas substantivas de investigação; à medida que o conceito se refina analiticamente por meio de sua integração a outros conceitos, a teoria cresce em profundidade e poder explicativo; e, por último, o conceito deve ser capaz de explicar as variações, casos contraditórios ou alternativos, ou seja, quando variam as condições, a explicação se mantém ainda que ajustada. Uma vez alcançada a saturação teórica das categorias, prossegue-se com revisão, ordenação e a integração dos códigos relacionados às categorias ${ }^{(7)}$. A etapa final do processo de geração da TFD é a integração que corresponde à organização cumulativa (ou articulação) dos componentes da teoria. As categorias e propriedades são ordenadas por similaridade, conexões e ordenamentos conceituais. Assim, as categorias relevantes que emergiram dos dados ficaram refletidas na análise conceitual ${ }^{(8)}$.

\section{RESULTADOS}

\section{Apresentando o Modelo de Sistema de Cuidados}

O Modelo Teórico de Sistema de Cuidados (Fig. 1), foi delimitado a partir do fenômeno central Construindo o Sistema Complexo de Cuidados determinado pelas categorias: Convivendo com a dinamicidade do sistema de cuidados e Organizando o sistema de cuidados a 
partir de múltiplas interaçoes. O modelo teórico conduz o debate para além dos determinismos estruturais e/ou reducionistas que empobrecem a complexidade do humano e se direciona ao princípio da transdisciplinaridade ${ }^{(9-10)}$, como possibilidade de convivência e de enfrentamento de uma lógica disciplinar fragmentada e rupturas com a linearidade de ler e interpretar o mundo, as coisas, as ações e as práticas de cuidado.

Construindo o sistema complexo de cuidado retrata um processo social, institucional, profissional e pessoal dinâmico e gradual que demanda tempo, atitude, comportamento e um esforço incansável dos profissionais na busca de novas formas de ação/interação, isto é, na busca do novo a partir daquilo que existe, do real. $\mathrm{O}$ novo, nessa perspectiva, só pode ser idealizado e perseguido a partir do velho, da consciência crítica da realidade e da vivência de subjetividades com capacidades e vontade de configurar um novo espaço. Num mundo de instabilidades, de inconformismos e desapontamentos, muitos profissionais da saúde, de modo especial os participantes desta pesquisa, refletem no fenômeno Construindo um sistema complexo de cuidados, um novo significado e/ou visualizam novas possibilidades de enfrentamento das práticas em saúde. As possibilidades de transformação no modo de cuidar podem ser evidenciadas nas categorias que emergiram do processo de investigação e, de forma mais concreta, por meio das falas dos entrevistados:

A questão do cuidado quebrado, fragmentado não faz mais sentido e o nosso trabalho aqui realmente é o cuidado, cuidado, num sistema de cuidado, porque cada profissional complementa o trabalho do outro, trabalha numa interdisciplinaridade, ou numa transdisciplinaridade, falando até num sentido mais amplo. (E6)

Ficar ao lado. É muito melhor ficar ao lado, ter o outro profissional como seu parceiro no sentido de somar. O trabalho se torna mais agradável, mais prazeroso. (E1)
Na categoria, Organizando o sistema de cuidados a partir de múltiplas interações, enfatizam-se as relações, atividades interligadas, trocas, totalidade, centralidade, conflitos, liderança, retroalimentação, entre outros processos, que dizem respeito aos movimentos que alimentam o sistema nas suas várias dimensões apresentadas nas falas abaixo:

O sistema tem muitas facetas, uma delas é trabalhar com vários profissionais... É a possibilidade de desenvolver relações, interações. E isso nem sempre é fácil. Já vivenciamos muitos conflitos. Hoje já sabemos lidar melhor com as diferenças de cada um na equipe. (E4)

O cuidado pra ser eficiente depende de uma rede de relações. $A$ rede é como se fosse uma cadeia, é como se fosse teia de aranha (E3). E isso só é possivel se na equipe de trabalho há interação, você começa a enxergar o outro de forma diferente. Isso é muito importante. (E6)

A categoria Convivendo com a dinamicidade do sistema de cuidados resulta das desorganizações, seguidas de reorganizações internas, sendo representada pelas subcategorias (Experenciando Mudanças, Identificando conflitos e Determinando significados do sistema de cuidados) definidas nas falas abaixo:

Nós já vemos um avanço no sentido de que um profissional procura olhar o outro, notar e achar importante a presença do outro, a participação, a contribuição que aquele outro tra\%. (E1)

Trabalhávamos de forma individualizada, na verdade acontecia uma fragmentação muito grande, e até uma competitividade permeava isso aí. Então hoje a gente já passeia pelos diversos, pelas diversas profissões com essa conscientização realmente, porque a gente não pode cuidar integralmente do outro de forma individualizada. (E 12)

Um cuidado integral, um cuidado amplo, ele é enriquecedor

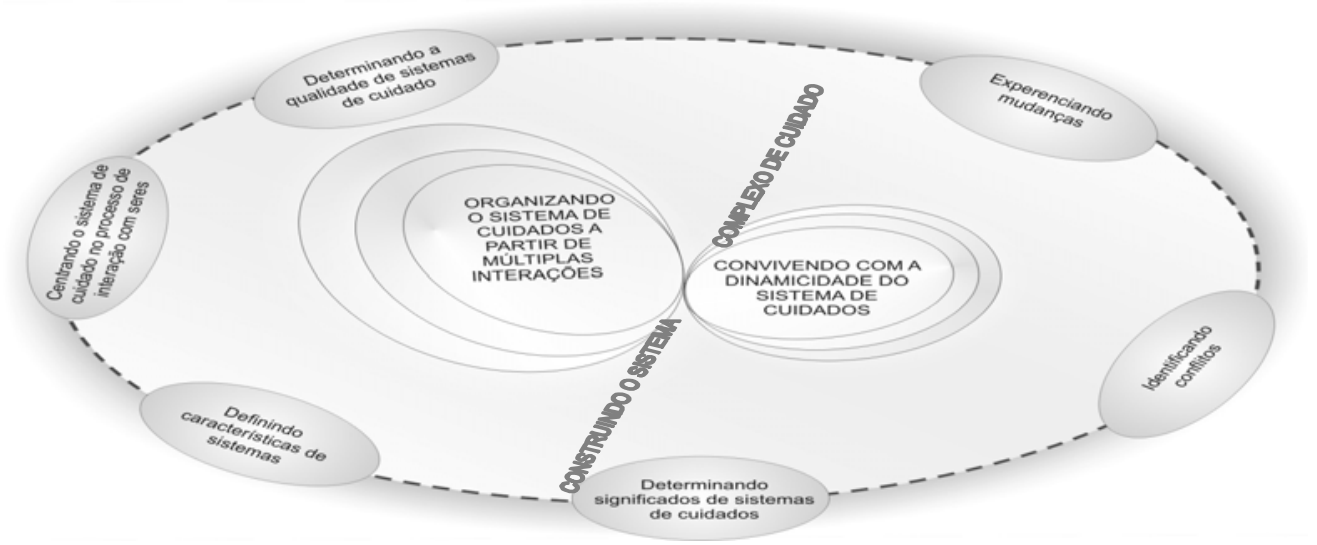

Figura 1. Modelo teórico de Sistema de Cuidados 
para todos os profissionais. Olha o que en aprendi com a fonoaudiologia, com a terapia ocupacional, com a psicologia, quando esse cuidado não é estanque, esse cuidado amplo é enriquecedor. Então a gente aprende muito, e os profissionais se enriquecem mutuamente e o cliente é o grande beneficiado. (E 3)

É mais isso não foi assim de repente, não. Levou tempo, muitos cursos, muitas vivências, pra gente compreender que esse cuidado era mais amplo, era mais adequado. (E 4)

A gente tem clareza de que trabalhamos a interdisciplinaridade, tendo o entendimento que os vários saberes, eles se interligam em algum momento, como se fosse uma flor que o centro é o cliente e as pétalas são os vários profissionais. Em algum momento essas pétalas se intercalam, se encontram. Então ai nesse encontro é a interdisciplinaridade. É mais ou menos assim que a gente entende, que a gente tenta trabalhar. Claro que enfrenta muitas barreiras, muitas dificuldades que são decorrentes daquela conotação que se tinha no passado de trabalhar só a multidisciplinaridade. (E 1)

Quando a gente fala de sistema de cuidados eu penso numa rede, numa rede multidisciplinar, numa rede hierarquizada, numa rede organizada para atender uma demanda (E 8). Sistema envolve muita coisa. Eu penso numa rede. .

Nesta perspectiva, o processo auto-organizador pode criar o radicalmente novo, ampliando a capacidade do sistema de cuidados de interagir com eventos aleatórios que o perturbam, assimilando-os e modificando a sua estrutura. O sistema de cuidados, portanto, caracterizase pela ambivalência e pelo paradoxo entre ordem e desordem, que conduz à passagem de um nível de organização a outro mais elevado, com novas propriedades, novas aptidões para assumir novas formas e maiores disposições relacionais. O grande avanço do cuidado, enquanto sistema complexo, parece incidir, sem dúvida, na dinamicidade e na multiplicidade de interações, nos aspectos parciais e na totalidade da realidade, do todo nas partes e das partes no todo. Assim, Definindo as características de sistema de cuidados, Centrando o sistema de cuidados no processo de interação com os seres humanos, Determinando a qualidade do sistema de cuidados, Experenciando mudanças no sistema de cuidados, Identificando conflitos no sistema de cuidados e Determinando significados de cuidado à saúde, enquanto subcategorias emergentes do processo de codificação, retratam a possibilidade de convivência e de enfrentamento de uma lógica disciplinar fragmentada presente no sistema de cuidados em saúde.

O modelo teórico Construindo o sistema complexo de cuidados potencializa, num sentido mais amplo, a capacidade do ser humano de se movimentar nos espaços organizacionais, construindo oportunidades de relações, interações e vivenciando o cuidado na ordem do seu potencial para demarcação e utilização dos diferentes espaços, ou seja, de dependência e/ou interdependência, de pertencimento e/ou privacidade. A convivência e a dinamicidade organizacional propiciam viver o aconchego das coisas simples, mesmo diante das tensões e riscos dos momentos mutantes, muitas vezes imprevisíveis e plenos de significados ${ }^{(11)}$. O significado de sistema de cuidados passa, necessariamente, pelo resgate dos pequenos/grandes eventos do dia-a-dia que tornam o ser humano único e especial nos diferentes espaços e situações em que se encontra, no sentido de prestar atendimento personalizado, voltado não para a doença, mas para o ser humano que adoece. Desse modo, o paradigma da complexidade ajudará o ser humano/ profissional a reconhecer a complexidade das realidades e revelará, não apenas as incertezas inerentes às próprias estruturas do conhecimento, mas também os "buracos negros de incerteza nas realidades presentes"(12). O ser humano, portanto, necessita estar inserido na complexidade das realidades, de forma crítica e consciente, para dar razões éticas e humanas às opções, tendo em vista que as abordagens teóricas que adota têm respaldo em concepções de tempo, de espaço, de contexto, por isso, nunca neutras e arbitrárias. Para compreender a dinamicidade organizacional, é imprescindível associar na dinâmica do cuidado, o tetragrama, caracterizado pelo entrelaçamento da ordem/ desordem, interação/organização. Assim, o sistema complexo de cuidados pode ser entendido como resultado das interações dinâmicas da desordem com os acomodamentos estáticos da ordem. Uma prática com a idéia de ser apenas desordem, também seria incapaz de conservar a novidade e garantir o desenvolvimento ${ }^{(12)}$. Para o enfrentamento dos problemas de saúde é necessário incorporar visões interdisciplinares e apoio intersetorial ${ }^{(13)}$, e a permanente interatividade e conectividade entre os profissionais, não só no sentido prático, mas principalmente no suporte relacional e multidimensional. Cada profissional, em seu campo específico de atuação, necessita desenvolver a sensibilização contínua, para tornar as relações humanas integradas e humanizadas ${ }^{(14)}$. Assim, Organizar o sistema de cuidados a partir de múltiplas interações e Conviver com a dinamicidade do sistema de cuidados representa, no sistema complexo de cuidados, um avanço ontológico e epistemológico no processo de superação das fronteiras disciplinares.

\section{Reconhecendo a complexidade do sistema de cuidados - Para Concluir}

Visualizar o sistema de cuidados em um modelo teórico explicativo elaborado a partir da TFD implica em reconhecer a complexidade do cuidado nas suas múltiplas dimensões e interações, transposta da realidade dos dados para níveis de abstração mais abrangentes, inclusive no âmbito das práticas de cuidado à vida e à saúde. A visão prática do fenômeno Construindo um sistema complexo 
de cuidados pressupõe atribuir um novo significado às práticas em saúde, em um olhar que compreende a complexidade das relações, das trocas e do próprio processo de cuidar em saúde, portanto, não pode ser apreendido como algo pronto, estático e/ou como um fim em si mesmo. Por caracterizar um movimento dinâmico e interativo, o fenômeno demanda um processo dialógico e reflexivo por parte dos profissionais da saúde, usuários, instituições e pesquisadores. Enquanto sistema dinâmico, o sistema de cuidados alcança a complexidade do ser humano, como sistema complexo. Construir o sistema complexo de cuidados, a partir da aquisição de saberes e competências, baseado na convicção de um novo referencial que dá conta das intersubjetividades, interações e dinamicidades, abre espaços para novas práticas em saúde que contemplem a sensibilidade e o engajamento social. Assim, a superação das fronteiras disciplinares proposta pelo cuidado, enquanto sistema complexo, vai além da simples interação e integração dos saberes, mas reflete e amplia as discussões acerca das múltiplas dimensões que envolvem o ser humano. Determinar o cuidado a partir de um processo interativo e dinâmico significa produzir um conhecimento complexo em saúde, a fim de alcançar a compreensão do cuidado da vida humana como um fenômeno que transcende a dimensão física e o enfoque assistencialista. Significa compreender o cuidado enquanto sistema que implica na construção de redes não lineares que atravessam as diferentes áreas do saber. Significa reconhecer a força criativa, dinâmica e transformadora do cuidado, presente nas mais variadas formas, dimensões e saberes do agir humano. Nesses termos, compreender a vida, o processo de ser saudável e o cuidado enquanto sistema, somente será possível pela integralidade, pela conectividade e pela concepção de um sistema mais plural e complexo. Defende-se, nessa perspectiva, a idéia de que novas formas de pensar, detalhadas nas subcategorias são fonte/fruto de um novo paradigma no modo assistencial. As características definidas no modelo teórico de sistema de cuidados aqui apresentado centram-se no processo de interação com os seres humanos e resulta na organização deste sistema. Por sua vez, este sistema se mantém no estar experenciando mudanças, identificando conflitos e determinando significados, caracterizando-o como processo dinâmico e como cenário de crescentes mudanças de atitudes e comportamentos no modo assistencial e relacional dos profissionais. Retrata a capacidade do ser humano de se movimentar nos espaços organizacionais, construindo oportunidades de relações, interações, e utilizando-se dos diferentes espaços: de dependência, de interdependência, de pertencimento e de privacidade. A organização do sistema de cuidados, portanto, deve sustentar-se na ação e no saber compartilhado dos vários profissionais e no trabalho em equipe que se expresse na cumplicidade da teia entre usuários/clientes e profissionais.

\section{REFERÊNCIAS}

1. Erdmann AL, Andrade SR, Mello ALSF, Horner V. Gestão das práticas de saúde na perspectiva do cuidado complexo. Texto \& Contexto Enfermagem. 2006; 15(3): 483-91.

2. Erdmann AL. Tendências dos sistemas organizacionais de enfermagem hospitalar: algumas contribuições. Florianópolis: UFSC; 1993. [Trabalho apresentado em Concurso para Professor Titular da Universidade Federal de Santa Catarina].

3. Morin E. O método 3: o conhecimento do conhecimento. Porto Alegre: Sulina; 1999.

4. Strauss A, Corbin J. Bases de la investigación cualitativa. Técnicas y procedimientos para desarrollar la teoría fundamentada. Medelin: Editorial Universidad de Antioquia, Facultad de Enfermería; 2002.

5. Strauss AL. Qualitative analysis for social scientists. Cambridge [Cambridgeshire]; New York: Cambridge University Press; 1987.

6. Glaser BG, Strauss AL. The discovery of grounded theory: strategies for qualitative research. New York: Aldine de Gruyter; c1967.

7. Glaser B. Grounded theory perspective III: theoretical coding. Mill Valley, Calif.: Sociology Press; 2005.

8. Glaser BG. Theoretical sensitivity: advances in the methodology of grounded theory. Mill Valley, Calif.: Sociology Press; c1978.

9. Gadotti M. Pedagogia da terra. São Paulo: Fundação Peirópolis; 2000.

10. Morin E. A cabeça bem-feita: repensar e reformar o pensamento. Rio de Janeiro: Bertrand Brasil; 2000.

11. Erdmann AL. Sistemas de cuidados de enfermagem. Pelotas: Universitária/UFPel; 1996.

12. Morin E. Ciência com consciência. 7a ed. Rio de Janeiro: Bertrand Brasil; 2003.

13. Pinto IC, Passeri IAG, Silva DS, Oliveira MM. (Re)organizando a sala de curativo do Centro de Saúde Escola da Faculdade de Medicina de Ribeirão Preto da Universidade de São Paulo. Acta Paul Enfermagem. 2005; 18(1): 89- 93.

14. Chiappin A. Psicologia das relações humanas no hospital. Porto Alegre: Globo; 1998. 